\title{
A Review of Recent Data in the Treatment of Gallbladder Cancer Naveed $S^{1}$, Qari $H^{2}$, Altaf $A^{3}$, Mahpara ${ }^{4}$
}

\begin{abstract}
:
Gallbladder cancer is now considered a distinct clinical entity, allowing for a separate analysis from that of other malignancies of the biliary tree. Symptoms related to a malignant tumor of the gallbladder include jaundice and abdominal pain, or a palpable abdominal mass that occurs in a late stage of the disease. The majority of patients with operable gallbladder cancer are diagnosed by cholecystectomy performed for presumed benign disease, mostly cholelithiasis, a clinical entity known as incidental gallbladder cancer. Given the poor prognosis if tumor invasion beyond the muscular layer and/or nodal metastasis is found, adjuvant treatments have been implemented, but few data are available to guide treatment decisions in this setting. For advanced disease, a multidisciplinary treatment approach including biliary drainage procedures and palliative support is needed in the management of this aggressive disease. Palliative chemotherapy with a combination of gemcitabine and cisplatin or oxaliplatin is the standard treatment based on the findings of two phase III trials that showed improved overall survival compared to single-agent chemotherapy and best supportive care. Several phase II studies have been reported investigating the role of targeted agents against EGFR, VEGF, HER2, and MEK. International collaboration to enhance our knowledge of gallbladder cancer should be encouraged.
\end{abstract}

Key Words: Gall bladder cancer, cholecystectomy, radical resection.

Bangladesh Journal of Medical Science Vol. 15 No. 03 July'16. Page : 320-325

\section{Introduction:}

Carcinoma of the gallbladder is an uncommon but lethal malignancy. It is the most frequent type of cancer of the biliary tract, and is now classified as a separate disease. Worldwide, gall bladder carcinoma is the sixth most common gastrointestinal cancer, with an annual incidence rate of 2.2 per 100,000 . However, its effect varies strikingly between different geographic areas with mortality rates being more than 10-times higher in Chile, where this disease represents the second most frequent cause of cancer death in women compared with the United States ${ }^{1}$.

The risk factors associated with GBC include cholelithiasis and other factors for chronic inflammation like Salmonella- or Helicobacterspecies carriers, female gender, obesity, smoking, and low socioeconomic status ${ }^{2}$. The vast majority $(65 \%$ to $90 \%$ ) of GBCs are adenocarcinomas, followed by squamous cell or adenosquamous ( $5 \%$ to $10 \%)$, and undifferentiated carcinomas (5\%). Tumor-registry data of over 2500 cases in the United States between 1985 and 1995 showed 5 years survivals of $15 \%, 5 \%$ and $1 \%$ for Stages II, III and IV patients, respectively. Approximately $70 \%$ of patients present with Stage III or Stage IV disease ${ }^{3}$. In tumors confined to the gallbladder wall, 5 years survival rates after resection only range from $10 \%$ to $30 \%{ }^{4,5,6}$. A recent study that analyzed data from the Netherland Cancer Registry of 3,917 patients reported an overall 5-year survival rate of $12 \%$, reflecting the aggressive nature of this disease characterized by extensive local and nodal invasion and early distant metastatic spread. Patients who had received surgery as part of their treatment presented a 5 -year survival rate of $19 \%$ to $26 \%$, with a tendency for the rate to improve over the last two decades ${ }^{7}$. Most patients with gallbladder carcinoma will present with more advanced disease

1. Shah Naveed, DNB Dept. of Surgical Oncology Mahavir Cancer Sansthan INDIA.

2. Hasina Qari, DNB Dept. Anesthesia IGIMS INDIA.

3. Asma Altaf, Srinagar.

4. Mahpara Srinagar.

Corresponds to: Shah Naveed, DNB Dept. of Surgical Oncology Mahavir Cancer Sansthan INDIA. Email: kingshahnaveed@yahoo.co.in. 
(i.e., adjacent-organ or metastatic involvement). Local invasion into surrounding tissue and liver is facilitated by the thin muscular gallbladder wall as well as continuum of the perimuscular connective tissue with the interlobular connective tissue of the liver ${ }^{8}$. Hepatic infiltration by gallbladder cancer (GBC) has been observed in $60-70 \%$ of patients on collective review and autopsy series ${ }^{9}$. Lymphatic spread by GBC is also common. Overall regional nodal involvement has been reported in $40-80 \%$ of patients ${ }^{9}$. For patients with T2 lesions (confined to the gallbladder wall), the incidence of nodal metastases ranges from $40 \%$ to $62 \%$ respectively $10,11,12,13$. When disease invades the covering serosa or adjacent organs, nodal metastases rates rise to $70-80 \%$ respectively ${ }^{12,13}$. The primary draining nodal groups are along the cystic and common bile ducts. Retrograde spread to hilar nodes can occur, particularly in more advanced disease ${ }^{14}$. Secondary spread occurs to the pancreaticoduodenal nodes and later to the periaortic nodes, both of which usually go undisected, even in more radical procedures. With either lymph node involvement or hepatic infiltration (Stage III/IV), prognosis is poor, with reported 5 years survivals of $5 \%$ or less ${ }^{4}$.

The most important prognostic factors are depth of invasion into the gallbladder wall and the presence of lymph node or distant organ metastasis: the revised tumor, node, metastasis staging system has proven to be useful when assessing prognosis at diagnosis. In the curative setting, R0 resection is the most important prognostic factor.

\section{Management of resectable gallbladder cancer}

Surgery is the only curative treatment for patients with GBC. Most of the resectable GBC cases are diagnosed incidentally. The finding of GBC after cholecystectomy for presumed benign disease (mainly cholelithiasis) varies from less than $1 \%$ to $3 \%$. In advert simple cholecystectomy is the most common surgical procedure for resection of primary carcinoma of the gallbladder ${ }^{3}$, as the diagnosis is not usually suspected pre-operatively. Most patients with incidental GBC are diagnosed based on the pathology report, and the oncological resection is done by a second surgical intervention. This procedure should be done by an experienced surgical team, and delayed oncological resection and referral to a cancer center or tertiary hospital is appropriate.

Even in early stage disease, positive margins after resection are common, given that the plane of dissection at simple cholecystectomy is sub serosal ${ }^{15}$. Therefore, many hepatobiliary surgeons advocate radical resection or re-resection (wedge resection of the gallbladder bed/hepatic resection, excision of regional nodes) in the treatment of Stage T2 or higher disease, although less than $10 \%$ of patients undergo such procedures ${ }^{3,16,17}$. Differing surgical series have reported that survival may be improved in patients with Stage T2N0 or higher disease by more radical operations 17, 18, 19, 20. Patients with microscopically positive margins after gross total resection have a statistically worse outcome compared with those with negative margins. Margin negative resection had a superior survival versus patients with positive margins ${ }^{21}$. However, we consider achieving marginnegative resection an important end point. The role of persistent local-regional disease contributing to the development of distant metastases is controversial, although in other disease sites, uncontrolled local disease appears to be a source of distant metastases ${ }^{22}$. Reports that describe patterns of failure after surgery are limited. Available data suggests that local-regional recurrence is common and ultimately leads to death, usually from complications of biliary obstruction and liver failure. Literature review indicates local recurrence occurs in up to $86 \%$ of patients after cholecystectomy. In long-term survivors after surgery, local recurrence rates remain high, even beyond 5 years ${ }^{23}$. A likely explanation for this finding is that occult nodal involvement is common and localized invasion of the liver is not recognized and respected. This high incidence of residual microscopic disease has been reported in autopsy series ${ }^{24}$. Even in patients treated with radical cholecystectomy local regional recurrence has been reported to be as high as $75 \%{ }^{23}$. A recent large study from Memorial Sloan-Kettering Cancer Center showed that in patients who undergo radical resection of GBC, $45 \%$ of relapse loco regionally.

For Tis and T1a tumors, the prognosis after simple cholecystectomy is very good and no further treatment is needed for these patients. Extended re-resection after an incidental T1b GBC diagnosis remains controversial. For tumors invading beyond the muscular layer $(\mathrm{T}>1)$ in addition to cholecystectomy, an oncological resection including limited hepatic resection and portal lymphadenectomy is the optimal surgical approach. A multicenter, multinational study, which included 148 patients, reported residual disease in $56.7 \%$ of $\mathrm{T} 2$ and in $77.3 \%$ of T3 incidental GBC after oncological re-resection ${ }^{25}$. The 5-year OS was $67.3 \%$ for $\mathrm{T} 2$ and $26.1 \%$ for $\mathrm{T} 3$ tumors: residual disease at re-resection and R0 surgery were strong prognostic factors. 
The R0 resection rate of this second surgical therapy varies widely, mostly because of findings of metastatic disease at relaparotomy, and rates as low as $38.8 \%$ have been reported ${ }^{26}$. If metastatic disease is confirmed, no further resection should be done. Patients with metastasis of nodes of the celiac axis or aorto-caval groove are considered unresectable.

The goal of surgery in gallbladder carcinoma is to achieve negative margins, and the resection can vary based on the extent of the disease. In locally advanced disease, where major hepatectomy and/or common bile duct excision would be necessary to achieve $\mathrm{R} 0$ resection, the potential benefit of these surgical interventions should be balanced against the increase in morbidity and the overall gloomy prognosis of these patients. A Surveillance, Epidemiology, and End Results Program (SEER) and Medicare data analysis, which included 1,899 patients with T2 and T3 tumors, showed a better survival for patients after radical resection or evaluation of more than three lymph nodes compared with cholecystectomy alone. The magnitude of this observation was clearer in T2 patients, and the overall 5-year survival rate for T3 patients with radical resection or lymphadenectomy was less than $20 \%{ }^{27}$. However, the proportion of patients who underwent radical resection/ hepatectomy was low (13.4\% for T2 and $18.2 \%$ for $\mathrm{T} 3$ cancers).

\section{Adjuvant Therapy}

Given the dismal prognosis of patients with GBC with $\mathrm{T} \geq 2$ and/or $\mathrm{N}+$ disease, even when resected with clear margins, many institutions have adopted adjuvant strategies. The National Comprehensive Cancer Network guidelines for GBC suggest adjuvant fluoropyrimidine chemoradiation or fluoropyrimidine, or gemcitabine chemotherapy, recognizing, however, that limited data exist to define a standard regimen. A recent systematic review and meta-analysis included six studies addressing the role of adjuvant treatments in $\mathrm{GBC}$, including one randomized trial, one database study using the SEER data, and four institutional series ${ }^{28}$. This analysis showed a nonsignificant improvement in survival comparing any adjuvant therapy with surgery alone . Node-positive and margin-positive (R1) patients derived the clearest survival benefit from the use of adjuvant therapies. This finding was also seen in a SEER-based study that had not been included in this meta-analysis. This study showed that with the exception of T1N0 patients, having received chemotherapy or radiation in a period of 6 months after surgery was associated with a better OS ${ }^{29}$.
Despite extended surgery with R0 resection and adjuvant chemoradiation, recurrence rates are high. We reported our experience with adjuvant radiation with or without 5fluorouracil-based chemosensitization after curative surgery: 23 of 44 patients presented recurrence (52\%), with $27 \%$ only local recurrence, $18 \%$ only distant recurrence, and $7 \%$ both local and distant recurrence at first relapse ${ }^{30}$. In another series we observed a better OS in patients who received adjuvant chemoradiation after extended surgery compared to simple cholecystectomy (5-year OS of $57 \%$ versus $27 \%, p=0.005$ ), confirming the importance of extended surgery as part of a curative treatment strategy ${ }^{31}$.

The results of two phase III, randomized controlled trials that investigate the role of adjuvant chemotherapy are awaited. Both trials recruit biliary tract cancers including GBC after macroscopically complete surgical resection. The United Kingdom trial (NCT00363584) that randomly assigned patients to eight cycles of capecitabine versus observation has completed enrollment. The French trial (NCT01313377) that randomly assigns patients to 12 biweekly cycles of gemcitabine plus oxaliplatin versus observation is recruiting, and inclusion of patients to this important trial should be encouraged. ${ }^{22,33}$.

Chemotherapy has also been used in gallbladder malignancies with little or no survival benefit demonstrated. The only prospective randomized study of chemotherapy in the treatment of gallbladder malignancies was reported by the Eastern Cooperative Oncology Group for inoperable patients. This study failed to demonstrate a survival benefit and reported an objective response rate of only $10 \%$ [34]. Other non-randomized trials using 5-FU chemotherapy alone or in combination with other chemotherapy agents infused systemically or locally have reported non-statistically significant improvements compared with surgery alone ${ }^{17,35}$. Park et al. ${ }^{36}$ in retrospective study reported that overall survival (OS) was not significantly different among the adjuvant therapies $(P=0.180)$, but disease-free survival (DFS) was $(P=$ $0.033)$. The 3 years OS and DFS from surgery alone, adjuvant chemotherapy and adjuvant radiotherapy and adjuvant concurrent chemo-radiotherapy were $64,78,36$ and $36 \%$ and 56, 69, 14 and $47 \%$, respectively. Overall, the chemotherapy group had a better prognosis, although there were no significant differences. He concluded from this study that adjuvant therapy is an effective treatment option for curative resected GBC. A large randomized controlled study is necessary to confirm the efficacy 
of adjuvant therapy. Newer adjuvant studies should be focused on gemcitabine-based chemotherapy or chemo-radiotherapy with molecular-based target agents.

\section{Surveillance}

After curative treatment, the patient should be followed with visits every 3 to 6 months for 2 to 5 years, although no data from trials are available to support this recommendation.

\section{Management Of Unresectable Gallbladder Cancer}

The Memorial Sloan Kettering Cancer Center reported their 10-year experience of GBC. Of 435 patients referred with the diagnosis of $\mathrm{GBC}, 159$ patients (36.6\%) presented as stage IV, and $70(16 \%)$ presented as localized mass or locally advanced, inoperable, and 206 (47.4\%) had been an incidental finding ${ }^{37}$. As shown in this series and other reports, more than half of the patients with GBC are diagnosed at an advanced stage, frequently with jaundice, abdominal pain, or a suspicious abdominal mass. Staging workout should include chest, abdominal, and pelvic CT scan and/or abdominal MRI. In patients presenting with jaundice, an endoscopic retrograde cholangiopancreatography can be diagnostic and therapeutic at the same time, if used for stent placement. Alternatively a magnetic resonance cholangiopancreatography can clarify the site and origin of obstruction.

Often, these patients present with cholangitis, and treatment with antibiotics and relief of the biliary obstruction can improve performance status to enable further therapies. Early inclusion of palliative care specialists in the treating team is recommended, given the aggressive course of this disease.

\section{Palliative Chemotherapy}

For unresectable or metastatic GBC, palliative chemotherapy is an option and has proven benefits in OS compared to best supportive care alone. The chemotherapy regimens supported by data from phase III trials are combinations of gemcitabine plus a platinum compound. The United Kingdom trial ABC-02 studied first-line palliative chemotherapy with gemcitabine with or without cisplatin in locally advanced or metastatic biliary tract cancer and enrolled 149 patients with GBC ${ }^{38}$. The addition of cisplatin to gemcitabine (GemCis) significantly improved OS in this group $(\mathrm{p}=<0.001)$. This regimen is considered the standard treatment for first-line chemotherapy in unresectable, recurrent, or metastatic GBC. This benefit has to be balanced against the toxicity of GemCis. The ABC-02 trial reported that $70 \%$ of the patients experienced grade 3 or 4 adverse events, with decreased neutrophil count, fatigue, and infection being the most frequently reported toxic effects. The Indian three-arm, phase III trial compared the combination of gemcitabine plus oxaliplatin (GemOx) to flurouracil plus folinic acid (FUFA), and to best supportive care ${ }^{39}$. With 82 patients enrolled, this study showed significantly longer OS in the GemOx group compared to best supportive care $(\mathrm{p}=0.01)$, but not in the FUFA group (HR $0.82,95 \%$ CI 0.45 to 1.51$)$ ( $p=0.053$ ). Grade 3 and 4 adverse events were reported in 19 of 26 patients $(73 \%)$ receiving the GemOx regimen, with myelosuppression being the most frequently observed, followed by transaminitis and neurotoxicity. It is noteworthy that the two phase III trials mentioned administered the chemotherapy for a maximum of 6 to 8 cycles. In the ABC-02 trial, only 26 of the 200 patients in the GemCis arm discontinued the treatment prematurely, mainly because of disease progression. With a planned treatment duration of 24 weeks, the median duration of therapy was 21 weeks. With the GemOx regimen, 5 of the 27 patients discontinued chemotherapy because of disease progression and 2 patients because of toxicity. Observation after 24 weeks of palliative chemotherapy is therefore a valid option.

No standard second-line chemotherapy regimen has been determined. The $\mathrm{ABC} 03$ trial plans to randomly assign patients with advanced biliary tract cancer to active symptom control versus active symptom control and combination chemotherapy with oxaliplatin, 5FU, and leucovorin after first-line gemcitabine and cisplatin ${ }^{40}$.

\section{Novel Agents and targeted therapies}

Common mutations reported in GBC are KRAS $(3 \%$ to $38 \%), E G F R$ ( $9 \%$ to $12 \%), B R A F(0 \%$ to $33 \%)$, and erbB2/HER2 (16\%) ${ }^{41}$. A phase III trial from Korea that assessed the efficacy of first-line treatment with gemcitabine and oxaliplatin with or without erlotinib for advanced biliary tract cancer included $31 \%$ of GBC patients. With 268 patients analyzed, the median progression-free survival was 5.8 months in the chemotherapy plus erlotinib group compared with 4.2 months for the chemotherapy alone group $(\mathrm{p}=0.087)$. For the GBC subgroup, a HR of 0.99 (95\% CI 0.63 to 1.58$)$ was reported. Median OS was 9.5 months for both groups ${ }^{42}$. Several phase II studies investigating other novel agents or combinations have been completed. All of these trials included GBC together with other biliary tract cancers or gastrointestinal malignancies. The interpretation of these results for the subgroup of GBC is therefore 
difficult.

Given the importance of R0 resection for cure, strategies to improve $\mathrm{R} 0$ resection after incidental GBC diagnosis or in locally advanced, potentially resectable patients should be explored. As in other gastrointestinal malignancies, preoperative therapies could be better tolerated than postoperative therapies, and thus assure a multimodality treatment in highrisk patients. The reduction of residual disease at the time of re-exploration could also improve prognosis. On the other hand, in patients who progress during preoperative treatment, representing early relapse, an aggressive intervention could be avoided. Additionally, trials with a neoadjuvant strategy could offer opportunities for the development of predictive markers that could guide personalized treatment decisions in the future.

International collaboration for trials conducted with GBC and that include patients from high-risk areas should be encouraged.

\section{Conclusion:}

Cholecystectomy is a valid surgical treatment for T1aN0 tumors. Stage II and III tumors should be treated with limited hepatic resection and portal lymphadenectomy, in addition to cholecystectomy if an $\mathrm{R} 0$ resection can be achieved. Adjuvant chemotherapy and/or radiation is an option for high-risk patients. Palliative chemotherapy with a combination of gemcitabine plus cisplatin or oxaliplatin for 6 to 8 cycles improves survival in patients with unresectable or metastatic gallbladder cancer. New treatment strategies should be explored to improve outcome in this challenging disease.

Carcinoma of the gallbladder remains a lethal malignancy. In the minority of patients that are resectable for cure, local-regional recurrence remains a major cause of morbidity and mortality. Adjuvant treatment with radiotherapy and chemotherapy is effective adjuvant therapy is necessary to improve treatment outcome of GBC following resection. Studies suggest that adjuvant chemoradiation therapy may be effective in the treatment of lymph node-positive T2/ T3 GBC after surgical resection. Further randomized controlled studies with a larger sample size and with a new chemotherapy regimen are needed to confirm similar therapeutic effects on other stage tumors.

\section{Conflict of interest: None}

\section{Reference:}

1. Ferlay J, Soerjomataram I, Ervik M, et al. GLOBOCAN 2012 v1.0, Cancer Incidence and Mortality Worldwide: IARC CancerBase No. 11. Lyon, France: International Agency for Research on Cancer; 2013 Accessed on December 29, 2013.

2. Stinton LM, Shaffer EA. Epidemiology of gallbladder disease: cholelithiasis and cancer. Gut andLiver2012;6:172-187.

3.Piehler JM, Crichlow RW. Primary carcinoma of the gallbladder. SurgGynecolObstet 1978;147:929-42.

4.Arnaud JP, Casa C, Georgeac C, Serra-Maudet V, Jacob JP, Ronceray J, et al. Primary carcinoma of the gallbladder - Review of 143 cases. Hepatogastroenterology 1995;42:811-5.

5.Houry S, Schlienger M, Huguier M, Lacaine F, Penne F, Laugier A. Gallbladder carcinoma: Role of radiation therapy. Br J Surg 1989;76:448-50.

6.Shani M, Hart J, Modan B. Cancer of the biliary system: A study of 445 cases. Br J Surg 1974;61:98-100.

7. Witjes CD, van den Akker SA, Visser O, et al. Gallbladder cancer in the Netherlands: incidence, treatment and survival patterns since 1989. Dig Surg. 2012;29:92-98.

8. Henson DE, Albores-Saavedra J, Corle D. Carcinoma of the gallbladder. Histologic types, stage of disease, grade, and survival rates. Cancer 1992;70:1493-7.

9. Sons HU, Borchard F, Joel BS. Carcinoma of the gallbladder: Autopsy findings in 287 cases and review of the literature. J SurgOncol 1985;28:199-206.

\section{[PUBMED]}

10. Fong Y, Kemeny N, Lawrence T. Cancer of the liver and biliary tree. In: DeVita V, Hellman S, Rosenberg S, editors. Cancer of the Liver and Biliary Tree. 6 th ed. Philadelphia: Lippincott, Williams and Wilkins; 2001. p. 1162203.

11. Shimada H, Endo I, Togo S, Nakano A, Izumi T, Nakagawara $\mathrm{G}$. The role of lymph node dissection in the treatment of gallbladder carcinoma. Cancer 1997;79:892-9.

12. Tsukada K, Kurosaki I, Uchida K, Shirai Y, Oohashi Y, Yokoyama N, et al. Lymph node spread from carcinoma of the gallbladder. Cancer 1997;80:661-7.

13. Ogura Y, Mizumoto R, Isaji S, Kusuda T, Matsuda S, Tabata M. Radical operations for carcinoma of the gallbladder: Present status in Japan. World J Surg 1991;15:337-43.

14. Fahim RB, McDonald JR, Richards JC, Ferris DO. Carcinoma of the gallbladder: A study of its modes of spread. Ann Surg 1962;156:114-24.

15. Shoup M, Fong Y. Surgical indications and extent of 
resection in gallbladder cancer. SurgOncolClin N Am 2002;11:985-94.

16. Gall FP, Köckerling F, Scheele J, Schneider C, Hohenberger W. Radical operations for carcinoma of the gallbladder: Present status in Germany. World J Surg 1991;15:328-36.

17. Morrow CE, Sutherland DE, Florack G, Eisenberg MM, Grage TB. Primary gallbladder carcinoma: Significance of subserosal lesions and results of aggressive surgical treatment and adjuvant chemotherapy. Surgery 1983;94:709-14.

18. de Aretxabala XA, Roa IS, Burgos LA, Araya JC, Villaseca MA, Silva JA. Curative resection in potentially resectabletumours of the gallbladder. Eur J Surg 1997;163:41926.

19. Muratore A, Polastri R, Bouzari H, Vergara V, Capussotti L. Radical surgery for gallbladder cancer: A worthwhile operation? Eur J SurgOncol 2000;26:160-3.

20. Donohue JH, Nagorney DM, Grant CS, Tsushima K, Ilstrup DM, Adson MA. Carcinoma of the gallbladder. Does radical resection improve outcome? Arch Surg 1990;125:237-41.

21. Kresl JJ, Schild SE, Henning GT, Gunderson LL, Donohue $\mathrm{J}$, Pitot $\mathrm{H}$, et al. Adjuvant external beam radiation therapy with concurrent chemotherapy in the management of gallbladder carcinoma. Int J RadiatOncolBiolPhys 2002;52:167-75.

22. Overgaard M, Hansen PS, Overgaard J, Rose C, Andersson $\mathrm{M}$, Bach F, et al. Postoperative radiotherapy in high-risk premenopausal women with breast cancer who receive adjuvant chemotherapy. Danish Breast Cancer Cooperative Group 82b Trial. N Engl J Med 1997;337:94955.

23. Cady B, McDonald J, Gunderson L. Cancer of the hepatobiliary system. In: DeVita VT, Hellman S, Rosenberg SA, editors. Cancer: principles and Practice of Oncology. Philadelphia: Lippincott; 1985. p. 741.

24. Vaittinen E. Carcinoma of the gall-bladder. A study of 390 cases diagnosed in Finland 1953-1967. Ann ChirGynaecolFennSuppl 1970;168:1-81.

25. Pawlik TM, Gleisner AL, Vigano L, el al. Incidence of finding residual disease for incidental gallbladder carcinoma: implications for re-resection. J Gastrointest Surg. 2007;11:1478-1487.

26. Butte JM, Matsuo K, Gönen M, et al. Gallbladder cancer: differences in presentation, surigcal treatment, and survival in patients treated at centers in three countries. J Am Coll Surg. 2011;212:50-61.

27. Mayo SC, Shore AD, Nathan H, et al. national trends in the management and survival of surgically managed gallbladder adenocarcinoma over 15 years: a populationbased analysis. J GastrointestSurg.2010;14:1578-1591.

28. Horgan AM, Amir E, Walter T, et al. Adjuvant therapy in the treatment of biliary cancer: a systematic review and meta-analysis. JClinOncol.2012;30:1934-1940.

29. Wang SJ, Lemieux A, Kalpathy-Cramer J, et al. Nomogram for predicting the benefit of adjuvant chemora- diotherapy for resected gallbladder Cancer.JClinOncol. 2011;29:4627-4632.

30. Müller B, Sola JA, Carcamo M, et al. Adjuvant chemoradiation for resected gallbladder cancer: Treatment strategies for one of the leading causes of cancer death in Chilean women. IndianJCancer2013;50:184-188.

31. González ME, Giannini OH, González P, et al. Adjuvant radio-chemotherapy after extended or simple cholecystectomy in gallbladder cancer. ClinTranslOncol. 2011;13:480-484.

32. NCT00363584. Capecitabine or Observation After Surgery in Treating Patients With Biliary Tract Cancer.http:// clinicaltrials.gov/ct2/show/NCT00363584?term=NCT00 363584\&rank=1. Accessed January 19, 2014.

33. NCT01313377. Gemcitabine Hydrochloride and Oxaliplatin or Observation in Treating Patients With Biliary Tract Cancer That Has Been Removed by Surgery. http:// clinicaltrials.gov/ct2/show/NCT01313377?term=NCT01 313377\&rank=1. Accessed January 19, 2014.

34.Falkson G, MacIntyre JM, Moertel CG. Eastern Cooperative Oncology Group experience with chemotherapy for inoperable gallbladder and bile duct cancer. Cancer 1984;54:965-9.

35.Macdonald JS, Smalley S, Benedetti J. Postoperative combined radiation and chemotherapy improves diseasefreesurvival (DFS) and overall survival (OS) in resected adeno carcinoma of the stomach and G.E. junction. Results of Intergroup Study INT-0116 (SWOG 9008). Proceedings of American Society of Clinical Oncology, Plenary $1 \mathrm{a} ; 2000$. p. 19.

36.Park HS, Lim JY, Yoon DS, Park JS, Lee DK, Lee SJ, et al. Outcome of adjuvant therapy for gallbladder cancer. Oncology 2010;79:168-73.

37. Duffy A, Capanu M, Abou-Alfa GK, et al. Gallbladder Cancer (GBC): 10-Year experience at Memorial Sloan-Kettering Cancer Centre (MSKCC). J SurgOncol. 2008;98:485-489.

38. Valle J, Wasan H, Palmer $\mathrm{DH}$, et al. Cisplatin plus gemcitabine versus gemcitabine for biliarytractcancer. NEnglJMed.2010;362:1273-1281.

39. Sharma A, Dwary AD, Mohanti BK, et al. Best supportive care compared with chemotherapy for unresectable gall bladder cancer: a randomized controlled study. J ClinOncol. 2010;28:4581-4586.

40. NCT01926236. Active Symptom Control Alone or With mFOLFOX Chemotherapy for Locally Advanced/ Metastatic Biliary Tract Cancers (ABC06). http://clinicaltrials. gov/ct2/show/NCT01926236?term=NCT01926236\&ra $\mathrm{nk}=1$. Accessed January 19, 2014.

41. Hezel AF, Deshpande V, and Zhu AX. Genetics of biliary tract cancers and emerging targetedtherapies. JClinOncol.2010;28:3531-3540.

42. Lee J, Park SH, Chang HM, et al. Gemcitabine and oxaliplatin with or without erlotinib in advanced biliary-tract cancer: A multicentre, open-label, randomised, phase 3 study. Lancet Oncol.2012;13:181-188. 